\title{
Los bots como servicio de noticias y de conectividad emocional con las audiencias. El caso de Politibot ${ }^{11}$
}

\section{Bots as a news service and its emotional connectivity with the audience. The case of Politibot}

\author{
Hada M. Sánchez Gonzales. Universidad de Sevilla (misago@us.es) \\ María Sánchez González. Universidad de Málaga/UNIA (m.sanchezgonzalez@uma.es)
}

Recibido: 14/09/2017 - Aceptado: 03/10/2017

\section{Resumen:}

Es perceptible que los usuarios se hallan conectados de forma permanente y ubicua a la red a través de sus dispositivos móviles. El periodismo cuenta con herramientas que, usando inteligencia artificial y a través de sistemas de mensajería instantánea, permiten remitirles información filtrada y personalizada. Son los llamados bots o aplicaciones conversacionales. Mientras los grandes medios están desarrollando sus propias aplicaciones vía Messenger o Telegram, vienen surgiendo empresas que ofrecen servicios de este tipo, como Politibot, lanzada durante la campaña electoral de junio de 2016 en España y que, un mes después, aglutinaba 8.400 suscriptores. La presente investigación analiza desde la perspectiva de las audiencias hasta qué punto los usuarios diferencian y valoran las noticias de los bots. Se aplican, entre otras técnicas, el análisis documental, observación directa, entrevista y ficha de análisis. Los resultados constatan que la calidad informativa (73,1\% de las respuestas) y la conexión emocional con la información recibida $(26,8 \%)$ son las principales fortalezas de estas fórmulas que se erigen, así, en una oportunidad para el periodismo.

\section{Palabras clave:}

Periodismo robot; innovación; audiencias; redes sociales; dispositivos móviles; inteligencia artificial.

\begin{abstract}
:
It is evident that users are permanently and ubiquitously connected to the network through their mobile devices. As a consequence, they can access to an extraordinary quantity of information. Journalism has tools that, by using artificial intelligence and instant messaging systems, send filtered and personalized information to users. These systems are the so called chat bots or conversational applications. While the big media companies are developing their own applications via Messenger or Telegram, there also exist other companies that provide this type of services. That is the case of Politibot, a service that was launched during the June 2016 election campaign in Spain and which had gained 8,400 subscribers a month later. This paper analyzes this case in order to determine the way audiences perceive and consider bots news. To do so, we apply, among other techniques, observation of Politibots publications via Telegram, survey of users and analysis of social media feedback. We aim to answer questions such as how users differentiate and value bots news, how conversation in these platforms is produced, and how to collect more reliable data from them. Results show that information quality (73.1\% of answers) and the emotional connection they establish with the tool (26, 8\%) are the main potential of these formulas, which are becoming in an opportunity for journalism.
\end{abstract}

\section{Keywords:}

Robot journalism; innovation; audiences; social networks; mobile devices; artificial intelligence.

1 Se enmarca en el proyecto I+D+i “La influencia de la audiencia en la innovación periodística y gestión dela participación: riesgos y oportunidades” (CSO2015-64955-C4-3-R-(MINECO/FEDER). 


\section{Introducción}

\subsection{El Periodismo en la era de conexión permanentemente y ubicua}

La llamada web 2.0 que definiera O’Reilly, caracterizada por la participación ciudadana y la eclosión de los llamados social media, ha dejado paso en los últimos años a un nuevo concepto de web, acuñado por el mismo autor junto al periodista Batelle (2009) que combina el mundo virtual con el mundo real. Es la llamada web al cuadrado o squared web. La web sigue siendo social, pero ahora a los medios sociales se suman nuevas tecnologías como los smartphones, los sensores o filtrado de datos, y nuevas formas de consumo, como el uso constante y masivo de redes sociales a través fundamentalmente de dispositivos móviles ${ }^{12}$. Unas prácticas que generan una ingente cantidad de datos que, en la era de la macroinformación y el big data, son procesados y analizados de forma constante con el objetivo de generar conocimiento, detectar tendencias o incluso realizar prospectiva.

En el ámbito del periodismo esta sobreabundancia informativa ha provocado el surgimiento bien de medios y aplicaciones orientadas a la gestión de la información y la curación de contenidos, bien de narrativas online como la visualización de la información u otros contenidos interactivos multimedia. Muchos medios han apostado por estas innovaciones como parte de su estrategia digital. Su desarrollo se produce, con frecuencia, a través de los llamados labs, que cuentan con equipos de periodistas y desarrolladores informáticos. Es el caso, en España, del Laboratorio de Innovación Audiovisual de RTVE, El Confidencial Lab o Vocento Medialab, entre otros (Salaverría, 2015: 397-404). Se busca con ello, además de facilitar la comprensión de asuntos complejos, atraer nuevos perfiles de lectores a las webs de los cibermedios y fidelizar a unas audiencias que, en la era de la sociedad líquida, siguiendo la idea del sociólogo Baumman (2002), cuentan en el ecosistema digital con múltiples fuentes, tradicionales y ciudadanas para informarse.

Pero el desarrollo de contenidos digitales interactivos online, como los citados, no es la única innovación que viene produciéndose en pro de atraer tráfico online y del engagement de los usuarios en los medios. Así, en los últimos años son muchos quienes vienen usando, por ejemplo, dispositivos móviles como vía de distribución de noticias e interacción, a través de apps propias o de ediciones webs adaptadas y, más tarde, de redes sociales como Whatsapp. Sin embargo, salvo determinados proyectos innovadores, estas prácticas de periodismo móvil han venido siendo facsimilares, en cuanto a contenidos y a las posibilidades de participación del periodismo digital convencional (Sánchez, 2011). Si Whatsapp y similares han venido siendo utilizados fundamentalmente como mero canal de alertas o resumen de noticias destacadas, generando tráfico de forma ubicua y permanente a los websites de los medios, la irrupción de los chatbots que operan a

12 Así, por ejemplo, el informe "La Sociedad de la Información en España de 2016" (VV. AA., 2016) apunta el móvil como el principal dispositivo a través del cual los españoles acceden a Internet, por delante del ordenador, con un $88,3 \%$ de usuarios, 5,9 puntos más que en 2014. 
través de estas y otras apps como sistemas inteligentes que permiten personalizar la remisión de información y la interacción con los usuarios, abre paso a nuevas posibilidades que los medios comienzan a experimentar.

En este trabajo pretendemos dar a conocer hasta qué punto los usuarios diferencian y valoran las noticias de los llamados bots, como nueva generación de periodismo móvil robot que potencia el consumo personalizado de noticias y la participación de las audiencias con un tono y unos recursos que, próximos a la idea de conversar con un amigo, buscan, además, su conectividad emocional. ¿¿Cómo es la conversación que se genera entre usuarios y bots?, ¿cuál es la opinión de los usuarios?, ¿̇supone una oportunidad para el periodismo, en cuanto a su potencial para la fidelización y captación de nuevos perfiles de usuarios? Son algunas de las cuestiones que intentamos dar respuesta en la presente investigación.

Para obtener un diagnóstico sobre los bots como servicio de noticias y la conexión con la audiencia en Telegram, nos planteamos como objetivos: 1. Dar a conocer el servicio que ofrece Politibot para informar sobre la actualidad. 2. Detectar cómo es la conversación entre Politibot y la audiencia y cuál es la opinión de los usuarios.

Los análisis reseñados en torno al periodismo robot son muy recientes (los más antiguos, de 2012) y bien proporcionan una visión exploratoria y global del fenómeno, recogiendo casos significativos en distintos medios y apuntando sus implicaciones para el ejercicio de la profesión periodística (Thurman et al., 2017; Clewall, 2014) o para las empresas que apuestan por estas fórmulas, o bien recogen las características, desde el punto de vista tecnológico de las aplicaciones (Dörr, 2015), o las clasifican atendiendo al uso que hacen los medios de las mismas (Lokot y Diakopoulos, 2015). Sin embargo, apenas se ha abordado desde la perspectiva de las audiencias, y menos aún en lo que respecta a los chatbots como herramientas cuyo potencial reside no solo en que suministran información personalizada a éstas sino en que lo hacen a través de un discurso cercano y de unas interacciones con las que buscan generar emociones y, con ello, fidelizarlas

\subsection{La robótica al servicio de la innovación periodística}

\subsubsection{La máquina de las noticias: casos pioneros de periodismo robot}

Al igual que en otros campos, la robótica y los sistemas de inteligencia artificial tienen su aplicación en el periodismo y han abierto la posibilidad a un nuevo mercado en la forma de producción y consumo de la información. Son las llamadas newsbots, aplicaciones de software diseñadas para recoger noticias publicadas para su consumo, de acuerdo con el criterio que el usuario definió. Según recoge Lemelshtrich en The New Word of Transitioned Media (2015), este Periodismo Robot se centraba en su inicio en dos pilares fundamentales:

"The computer software that automatically extract new knowledge from huge data silos employing the new 'social Physics' concept; algorithms that automatically convert this knowledge into readable stories without human involvement. Besides the great potential saving in labor costs, these robot journalists seldom miss facts, 
if programed correctly, are never tired and if programed objectively-are free of personal bias. Data silo managers of the media organizations and the AI narrative software engineers may become the key employees of the organizations. The human journalists, considering labor and other costs, may become obsolete".

Si la prospección y la analítica de datos, mediante sistemas informáticos, han estado ayudando a los periodistas desde hace décadas a construir historias, ahora la novedad es que mediante nuevos programas de Inteligencia Artificial se pueden recopilar hechos y redactarlas en un tiempo récord.

En Europa uno de los medios pioneros ha sido el británico The Guardian. Ya en 2010, interesado por los bots, recogía dos proyectos de automatización de noticias deportivas que permitían obtener estadísticas de los juegos e información histórica del equipo y los jugadores. Además, combinaban estos datos con frases pre-hechas y conectores para componer historias (Bunz, 2010). Un año después lanzaría una app que permitía automatizar búsqueda de noticias de actualidad vía Twitter (González, 2011) y en 2014 el llamado “Guarbot” (Gani y Haddou, 2014), un programa que completaba la información financiera con datos complejos, evitando a los periodistas realizar la tarea. Ese mismo año llegaría incluso a publicar un periódico en papel gratuito y de periodicidad mensual, con edición estadounidense y luego británica, cuya selección de temas se realizaba mediante sistemas de inteligencia artificial (233grados/La Información, 2014).

En paralelo, la revista Forbes trabajaba con la empresa Narrative Science, en una especie de periodista virtual que escribía sobre asuntos económicos y deportivos. De igual forma, la agencia de noticias Associated Press, pionera en el uso de estas aplicaciones inteligentes para producir noticias en Estados Unidos sobre temáticas tales como ligas menores de baseball, además de economía y tecnología. De las 300 historias que se producían de forma manual antes de su implementación, hacia finales de 2014, la agencia había pasado a ofrecer 4.000 historias, que se publicaban, en su caso, con el anuncio "Esta historia fue generada automáticamente por Automated Insights usando datos de Zacks Investment Research".

También en 2014, el periódico francés Le Monde utilizaba la inteligencia artificial de Data2Content para generar 30.000 micronoticias sobre resultados electorales en pequeños municipios y comarcas, un contenido que, como apuntaba el director adjunto del diario, habría sido imposible generar por sus profesionales y que aporta valor al medio (Sainz/Vocento Media Lab, 2016). Aparte de la actualidad política y económica, que ha sido objeto también de otros proyectos más recientes desarrollados por medios como NBC News (Morán, 2016), el periodismo de servicios, entre otros, también encuentran en los bots nuevas posibilidades. Es el caso por ejemplo, de Los Ángeles Times, donde Ken Schwencke, periodista y programador, desarrollaba en marzo de 2014 un algoritmo, "Quakebot" que se aplicaría a la creación de sistemas de alertas y noticias sobre terremotos de California.

Los propios social media han sido escritos también con ayuda de sistemas informáticos: baste reseñar el curioso caso del sueco Severker Johansson quien, de acuerdo a las estadísticas de la propia Wikipedia, es el autor más prolijo de este medio 
social, con 2,7 millones de artículos en 2014 (Emmerentze, 2014). Su secreto está en el uso de un software que bautizó como "Lsjbot", que extrae información de diversas bases de datos online y la resume en un borrador que luego revisa.

\subsection{Los chatbots: características, ejemplos y posibles aplicaciones en periodismo}

Pero el potencial de los bots, de acuerdo a proyectos más recientes, está, más que en la producción de noticias, en su distribución personalizada y en su poder conversador con las audiencias, mediante aplicaciones de mensajería instantánea. Son los llamados chatbots o bots conversacionales, aplicaciones que distribuyen contenido personalizado, a modo de charlas con los usuarios y que pueden, en cierta forma, considerarse una segunda generación de newsbots (Al Johri et al., 2016).

Es el caso de "Purple", una app que usa bots para poner en marcha un periodismo cercano, que en lugar de publicar contenido en la web, hace llegar historias a través del móvil, y que ha llegado a conformarse en un auténtico medio. La clave de su éxito, además de su propio tono conversacional, está de acuerdo a su cofundadora, Rebecca Harris, en que se dirige a donde están los usuarios.

Los tradicionales sistemas de alertas de noticias genéricos que los medios vienen implementando desde estas aplicaciones de mensajería han dado paso a sistemas inteligentes, mediante bots, usando casi siempre aplicaciones de mensajería instantánea que, como Telegram, Whatsapp o incluso Messenger de Facebook, cuentan ya con un elevado nivel de penetración entre la población de diversos países, incluyendo América Latina. Y aunque de momento la mayoría de proyectos están aún en fase de experimentación, en 2016 esta tecnología ha permitido cubrir ampliamente y en tiempo real, entre otros, grandes eventos como los Juegos Olímpicos de Brasil o las elecciones estadounidenses. The Washington Post, Forbes, The Huffington Post, BBC, CNN o Wall Street Journal, entre otros, cuentan ya con los suyos (Sainz/ Vocento Media Lab, 2016).

También en España los bots han comenzado a implantarse, sobre todo a mediados de 2016, y son ya varios los medios que cuentan con apps de este tipo. Así, la edición digital de El País, que en 2014 ya había lanzado la web Verne, basada en la localización y distribución de noticias de la Red (El País, 2010), presentó en verano de 2016 un bot vía Facebook para facilitar el consumo de noticias personalizadas desde el móvil en vacaciones (El País, 2016). De forma similar, eldiario.es viene usando, desde marzo de 2016 y tras un testeo durante las últimas elecciones generales en España, Telegram para su canal. Así explicaban la elección y el funcionamiento de esta herramienta:

"Por si no sabes lo que es Telegram, es como Whatsapp pero con el icono en azul. En realidad no es exactamente como Whatsapp, porque tiene lo que llaman “Canales", que es cómo vamos a mandar estos mensajes. Si Whatsapp tuviera una funcionalidad similar, la usaríamos sin dudarlo, pero no la tiene. En eldiario.es conocemos Telegram desde hace bastante tiempo, y de hecho en 2014 entrevistamos a su creador, Pavel Durov, usando la propia app para hacer dicha entrevista. Miles de personas compartieron con nosotros en Telegram la última 
jornada electoral del 20D. Vamos a seguir experimentando juntos y a vivir la actualidad de otra manera. Únete en telegram.me/eldiarioes".

El desarrollo de chatbots ha tenido lugar en el seno de los citados Labs, como sucede con el de Vocento, que ha lanzó a inicios de 2017 Infosky, experimento de periodismo robot para la temporada de nieve (Pérez, 2017). En sus primeros seis meses de trayectoria, se testeó a través del portal para jóvenes Eslang a modo de celebración de sus 100.000 seguidores, y como "forma lúdica y rápida" de acceder a su contenido de forma personalizada. En este caso ofrece versiones para Messenger y Telegram con las que los usuarios pueden seleccionar mediante palabras clave temas que les puedan interesar, como política, sexo, series o virales, y con las que, además, pueden interactuar participando en tests o trivials e, incluso, "mandar a paseo al robot (si uno logra sortear sus ingeniosas respuestas)" (Sainz, M./ Vocento Media Lab, 2016). Unas apps que como apuntan los profesionales de Vocento Labs, están en desarrollo continuo, atendiendo a las respuestas de los usuarios.

Como señalaba el periodista Pau Llop (2016), si antes los medios invertían en crear y actualizar sus propias apps de noticias (Sánchez González, 2011; 2014), ahora han encontrado "desarrollos mucho más ágiles y efectivos" en estos bots que pueden, además, configurarse de forma más o menos sencilla y rápida (Pachico, 2016). Con estos botsellos, tal como escribía recientemente Eduardo Manchón (2016), un medio pasa a ser un contacto más de Whatsapp o Messenger, y el consumo de noticias y el diálogo con los medios se produce en lo que es ya un entorno de conversación natural y técnicamente familiar para los usuarios que podría, según algunas visiones, llegar incluso a sustituir a los buscadores como puerta de acceso natural a Internet.

La idea del daily me que acuñara, décadas atrás, Negroponte, parece estar haciéndose realidad con los bots. En el sentido de que éstos, que incluyen sistemas de filtrado por palabras clave o geolocalización, pueden servir para que los usuarios reciban contenidos según sus preferencias temáticas, su autor o los eventos que suceden a su alrededor. Pero más allá, en una especie de gamificación, también abren la posibilidad de interaccionar con los lectores y potenciar la participación ciudadana adjuntando desde emoticonos a archivos multimedia; o de ciudadanos o los propios periodistas, filtren información o comprueben su veracidad mediante el diálogo vía móvil.

Las aplicaciones móviles estrechan la relación entre el periodista y la fuente de información. "Permiten establecer un contacto directo con las personas y aportar un panorama diferente a la información publicada asiduamente en los medios". Un caso real de conexión emocional entre el periodista y la audiencia se dieron en las revueltas de Oriente Medio. Como ejemplo, podríamos citar la crónica periodística que, por medio de la sucesión de mensajes cortos, se publicaron en Twitter por el periodista Mohamed Abdelfattah con el nombre del usuario (@mfatta7) (Sánchez, Méndez, 2013: 138) “Gas lacrimógeno (21:12 hrs.); Me asfixio (21:13 hrs.); ¡Socorro! (21:24 hrs.); Me dieron fuerte paliza (21:27 hrs.); Me van a detener...”. 


\section{Metodología}

Diseñamos una metodología que se apoya en la utilización de técnicas cualitativas y cuantitativas para realizar, en primer lugar, el análisis documental de la literatura específica sobre los bots como servicios de noticias, y, en segundo lugar, recurrir al método de estudio de caso para medir y registrar "la conducta de las personas involucradas en el fenómeno estudiado" (Martínez, 2006:11) dando respuesta a dos de nuestras variables que tienen que ver con el qué y cómo se realiza el servicio de noticias y con el cómo se efectúa la conversación con la audiencia.

En primer lugar, para responder a la primera variable, hemos utilizado la técnica de observación directa e indirecta como estrategia del método científico (Fernández-Ballesteros, 2004). Como observadores participantes, hemos entrando en la aplicación para interactuar con el bot en un periodo comprendido desde el 9 de enero al 29 de marzo, con el fin de constatar cómo se ha realizado el servicio de noticias en la plataforma de mensajería instantánea. En segundo lugar, se ha procedido al análisis documental sobre Politibot por medio de sus políticas de uso y de la entrevista ${ }^{13}$ en profundidad realizada al periodista Eduardo Suárez, cofundador de Politibot. De esta forma, posibilitamos un estudio analítico en la investigación.

En tercer lugar, para dar respuesta a la segunda variable sobre la interactuación/conversación con la audiencia, la unidad de análisis, como objeto que se pretende estudiar, ha estado comprendida por las elecciones en Holanda que se celebraron el 15 de marzo de 2017. Empero, con el fin de asegurar la validez de los datos obtenidos, se tuvo en cuenta un margen entre 7 y 8 días antes y después, respectivamente, de la fecha señalada. Dicho esto, el espacio temporal de análisis se realizó desde el 8 al 23 de marzo.

Para la recogida de datos sobre el tratamiento informativo proporcionado por Politibot a los usuarios, por un lado, hemos utilizado la técnica de la entrevista semiestructurada a uno de sus creadores, al periodista Eduardo Suárez. La principal ventaja a la que nos enfrentamos fue la flexibilidad para obtener datos y profundizar en algún punto en concreto solicitando al entrevistado la aclaración respectiva. Al mismo tiempo, nos ha garantizado la recopilación de la información necesaria para nuestro estudio. Realizamos seis preguntas abiertas determinando de antemano la información relevante que queríamos conseguir. Empero, con la utilidad de desarrollar preguntas que vayan surgiendo en el curso de la entrevista.

Por otro lado, hemos recogido la opinión de los usuarios sobre la cobertura del bot en Telegram a través de Twitter. Aplicación que nos ha permitido suministrar datos de la conducta individual y colectiva de los usuarios y determinar cómo ha sido la interactuación que se ha establecido por medio de la conversación. La principal ventaja que nos ha ofrecido Twitter, como herramienta de investigación, ha sido el poder acceder a los datos, clasificarlos y seguir las conversaciones

13 Encuentro que se realizó en la Facultad de Comunicación de la Universidad de Sevilla y la organización del mismo estuvo a cargo del Laboratorio de Proyectos en Comunicación (LabProCom) de la citada institución académica. 
en función de la búsqueda, es decir, el nombre del usuario del bot en dicha aplicación @politibot. La técnica de registro ha sido la ficha de análisis en función de las variables anteriormente citadas y, teniendo en cuenta los siguientes indicadores:

- Número de respuestas del usuario a través de Twitter.

- Calidad del servicio de noticias del bot en relación a la información que distribuye, por ejemplo, nivel de información en la cobertura sobre un hecho (uso de material propio o de otros medios), difusión de la información con elementos ilustrativos (gráficos, imágenes), multimedia (podcast, vídeo y gifs). Se ha procesado la información considerando la escala muy satisfecho, satisfecho, normal, poco satisfecho e insatisfecho, según los comentarios de los usuarios.

- Interactuación cercana y próxima con la audiencia, como la inclusión de emotions, frases o palabras con fórmulas de cortesía en el lenguaje o empleo del lenguaje figurado teniendo en cuenta su tipología (metáfora, simil, hipérbole, personificación, atenuación e ironía) así como la presencia o ausencia de las emociones (Goleman, 1996) y (Bisquerra, 2000) positivas: alegría (entusiasmo, euforia, excitación, contento, deleite, diversión, placer, estremecimiento, gratificación, satisfacción, capricho, éxtasis, alivio, regocijo, diversión); humor (sonrisa, risa, carcajada, hilaridad): amor (afecto, cariño, ternura, simpatía, empatía, aceptación, cordialidad, confianza, amabilidad, afinidad, respeto, devoción, adoración, veneración, enamoramiento, agape, gratitud); felicidad (gozo, tranquilidad, paz interior, dicha, placidez, satisfacción, bienestar); emociones ambiguas (sorpresa, esperanza, compasión) y negativas: ira (rabia, cólera, rencor, odio, furia, indignación, resentimiento, aversión, exasperación, tensión, excitación, agitación, acritud, animadversión, animosidad, irritabilidad, hostilidad, violencia, enojo, celos, envidia, impotencia); miedo (temor, horror, pánico, terror, pavor, desasosiego, susto, fobia, ansiedad, aprensión, inquietud, incertidumbre); ansiedad (angustia, desesperación, inquietud, estrés, preocupación, anhelo, desazón, consternación, nerviosismo).

A la vista de lo expuesto, las múltiples fuentes de evidencia que hemos utilizado garantizan la validez de los resultados o principio de triangulación con el objetivo de obtener datos fidedignos propios del rigor científico.

\section{Resultados}

\subsection{Telegram y el caso de Politibot}

La demanda de las aplicaciones de mensajería instantánea en los dispositivos móviles es cada vez mayor. Plataformas móviles como Whatsapp, Line, Facebook Messenger, Telegram son utilizadas por la población española con mayor asiduidad. Según el último barómetro publicado por el Centro de Investigaciones Sociológicas (CIS, 2017), confirman que el 70,2\% de los españoles reconoce haber usado aplicaciones de mensajería instantánea en los últimos seis meses. Nos centramos en Telegram por su utilidad, aunque no sea utilizado en la misma medida que Whatsapp y Facebook Messenger en España. 
Telegram desde finales de 2015 empezó añadir canales en su aplicación. Esta herramienta sirve para transmitir mensajes públicos, es decir, tiene la función de distribuir información de interés para grandes audiencias. Los medios de comunicación han visto una nueva forma de acercarse a la audiencia con el criterio de ofrecerles las principales propuestas informativas acompañadas de un enlace e imagen para ampliar la información e incluso, el uso de audio de forma tímida. En España, son varios los casos de medios y agencias de noticias que han apostado por esta aplicación haciendo uso solo de los canales que ofrece el servicio de mensajería, como por ejemplo, la agencia de noticia Efe y medios de comunicación como: eldiario.es, El País, El Mundo, El Economista, El Español, El Periódico, El Confidencial, CTXT, Cambio16, Cuarto Poder, El Jueves, OKdiario, Público y Vozpópuli.

Empero, la aplicación no solo dispone de canales, sino que tiene incorporada la opción de bots como recurso para que cualquier persona o empresa pueda crear un sistema de interacción automática con los usuarios. En Telegram, es posible localizar bots que ofrecen distintos servicios de acuerdo a una temática específica, como por ejemplo, DoNotPay, creado por un joven de 19 años, Joshua Browder (Gibbs, 2016). Es conocido como el primer robot abogado que ayuda a las personas a recurrir las multas de los aparcamientos y sirve de guía en el proceso de apelación. El bot es todo un éxito por el número de peticiones que ha recibido y que ha ganado en los procesos de apelación a pesar de su corta existencia. Podemos citar otros ejemplos como: @ImageBot, información del tiempo; @CorreYvuela, compra de billetes; @icon8bot, aplicación de filtros para fotografías; @octopocket_bot, envío de dinero entre usuarios; @GifBot, envío de gifs animados; @andyrobot, permite practicar inglés y @triviabot para jugar al trivial.

\subsection{Servicio de noticias}

Politibot es el primer servicio de noticias en Telegram que ofrece a sus usuarios información política facilitando la interactuación. Fue lanzado el 9 de junio del 2016 en España cuando comenzó la campaña electoral del 26 de junio (26J) brindado a los suscriptores visualizaciones de datos y un análisis de los patrones probables de votación basado en estudios sociológicos. A finales del mes de su creación (junio), ya contaba con más de 8.400 suscriptores. Eduardo Suárez, uno de los periodistas creadores, señaló en "Sinercom16", networking entre proyectos universitarios y empresas, organizado por el Laboratorio de Proyectos de Comunicación (LabProCom) de la Universidad de Sevilla, que era necesario desde el principio agregar un valor añadido a la audiencia ofreciendo “información útil y personalizada” (Suárez, 2016). De ahí el éxito obtenido superando las expectativas de sus creadores.

\subsubsection{Política de uso y características principales}

Politibot se presenta como un bot que puede mantener conversación sobre diversos temas. Igualmente, puede hacer preguntas sobre la información de actualidad o pedir la opinión del usuario e, incluso, preguntarle por sus gustos a cambio 
de ofrecerle información de interés. Como se describe en sus políticas de uso: "lo que viene siendo una conversación de toda la vida, con la ventaja de que no tengo vida amorosa y no te voy a aburrir con ella” (Politibot, 2017).

Su equipo fundador está formado por un grupo interdisciplinar de profesionales constituido por siete personas, tres de ellos periodistas: María Ramírez, Eduardo Suárez y Martín González, dos desarrolladores informáticos: Juan Font, Miguel Gil y David Martín-Corral y dos politólogos: Jorge Galindo y Kiko Llaneras. Un grupo de personas que, según Eduardo Suárez, lo que les une es la pasión por la política y por contar las cosas de otra manera. "Esas dos pasiones nos llevaron a finales de mayo de este año a pensar en cómo cubrir de otra manera las elecciones generales”. Añade: "algunos de nosotros ni siquiera nos habíamos visto antes y trabajamos un poco a distancia y entonces cubrimos las elecciones durante tres semanas o tres semanas y media del 2 al 22 de Junio" (Suárez, 2016).

El bot, en un inicio, fue creado para informar sobre la actualidad política en España con las elecciones en 2016, sin embargo, actualmente, también informa sobre política internacional. Ha cubierto el referéndum del Brexit, las elecciones electorales en EE.UU. y el triunfo de Donald Trump, las elecciones en Holanda, el atentado perpetrado en Londres y las elecciones de Francia.

Politibot proporciona información a los usuarios a través de enlaces a noticias, blog o estudios que se relacionen con la respuesta de la audiencia. Asimismo, incluye gráficos, fotografías, gifs animados y audio a través de podcast producido junto a Cuonda.com, una start-up independiente en español y que busca abrir nuevas vías de difusión e ingresos al podcasting hispano. Lo mejor de todo es que, a través de establecer conversación con los usuarios, les proporciona información relevante.

También, puede tratar datos sobre "preferencias, gustos, ideología, situación laboral actual, profesión, estudios, intereses, etc." de la audiencia. Politibot, (2017) deja claro que no es obligatorio que el usuario conteste a todo lo que se le pregunta y puede cambiar de tema, si así lo desea. En el caso de que el usuario acepte expresamente, el bot utilizaría sus datos con la siguiente finalidad:

- Ofrecer productos y servicios a mitad de precio y considerando la calidad del mismo, según las preferencias del usuario.

- Uso de geolocalización para brindar productos y servicios al usuario, siempre y cuando la tenga habilitada. Podrá obtener ofertas de establecimientos ubicados de acuerdo al lugar en el que se encuentre.

- El bot preguntará al usuario la conformidad de la cesión de sus datos de contacto (correo electrónico y/o teléfono móvil) para facilitar la información a terceras empresas para el envío de ofertas personalizadas.

En relación a la rentabilidad de @Politi_bot, Eduardo Suárez (2016) desveló que: 
"La idea es intentar explorar en este negocio algo que los medios todavía no han explorado, como la posibilidad de vender servicios similares a Politibot a empresas, particulares e incluso a medios de comunicación que quieran comunicar de otra manera y para todo eso necesitamos inversión, hasta ahora lo que tenemos es una ayuda de Google que nos ha dado hace unos días dentro del fondo de innovación que tiene para medios en Europa y el hecho de que nos hayan dado esa ayuda nos hace pensar que en el campo pueda haber una oportunidad".

Está claro que el bot brinda una serie de servicios y productos al usuario sobre la actualidad política. También informa según el perfil del usuario y aceptación expresa del mismo sobre distintas ofertas, aunque si bien es cierto, actualmente, el bot todavía no ha generado dicha información, ni ingresos por ello.

\subsubsection{Conversación con la audiencia}

Se describe como una herramienta que envía información personalizada y automática a través de la conversación. De acuerdo con su política de uso dice: "solo iniciaré una conversación si te diriges a mí y quieres que hablemos" (Politibot, 2017). El bot inicia la conversación con un mensaje que puede ser una pregunta o una información. Luego, el usuario se encuentra con un menú con cuatro opciones que varían según la actualidad política. Los temas que puede sugerir son: Trump, España, Podcast e Inspírame. Las dos primeras corresponden a noticias internacionales y nacionales, respectivamente. El tercero de ellos, es el servicio de podcasting producido por Cuonda que ofrece el bot a los usuarios. Se trata de episodios que se publican de la mano de los periodistas María Ramírez y Eduardo Suárez junto a invitados especiales, según el contexto nacional o internacional de la actualidad política.

Finalmente, el cuarto y último, Inspírame, hace alusión a una frase que remite el bot de algún autor para motivar y/o sugerir algo. Como ejemplo, podemos citar el siguiente texto remitido por @Politi_bot (2017): “La libertad, Sancho, es uno de los más preciosos dones que a los hombres dieron los cielos; con ella no pueden igualarse los tesoros que encierran la tierra y el mar: por la libertad, así como por la honra, se puede y debe aventurar la vida” (Miguel de Cervantes). Con las cuatro opciones fundamentales, antes mencionadas, es posible iniciar la interacción con el bot.

Igualmente, el bot ofrece información personalizada al usuario identificándole con un mensaje cercano y empático. Para describir cómo es la conversación que se establece entre el usuario y Politibot, nos hemos apoyado en el dialogo identitario que se asienta en el paradigma identitario de proximidad (Sánchez y Sobrados, 2008) porque considera la conducta adaptativa del usuario que se encuentra determinada por los sinequismos (principio de continuidad), por el proceso del paradigma códico del periodismo emotivo (considera el código social y los repertorios de signos concibiendo los diferentes estados del usuario en relación con sus vivencias y a través de la acción íntegra entre emisor y receptor) y por procesos inferenciales. 
Citaremos el caso del atentado de Londres ocurrido el 23 de marzo de 2017. El diálogo entre Politibot y el usuario se inicia con un breve saludo y las necesarias presentaciones, luego el bot informa de lo ocurrido a la vez que obtiene información del usuario sobre el grado de conocimiento del tema en cuestión, como puede apreciarse en el siguiente cuadro.

Cuadro 9. Politibot y el atentado de Londres

\begin{tabular}{|c|c|}
\hline \multicolumn{2}{|r|}{ Conversación } \\
\hline$@$ @olitibot & Buenas noches, human@.Ha sido una tarde trágica en el centro de Londres. Aquí te cuento lo esencial. \\
\hline @usuario & Vale \\
\hline @politibot & $\begin{array}{l}\text { Un hombre ha arrollado a varias personas en el puente de Westminster. Luego ha apuñalado a uno de los } \\
\text { policías que custodian el Parlamento británico. Cuatro personas han muerto durante el ataque, incluidos el } \\
\text { policía y el agresor. ¿Conoces la zona? }\end{array}$ \\
\hline @usuario & Sí \\
\hline @politibot & $\begin{array}{l}\text { Este mapa de la BBC explica muy bien la secuencia de los hechos y el entorno donde han ocurrido. } i \text { Te cuento } \\
\text { los datos esenciales? }\end{array}$ \\
\hline @usuario & Vale \\
\hline$@$ @politibot & $\begin{array}{l}\text { LO QUE SABEMOS DEL ATAQUE DE LONDRES } \\
\text { - Un hombre ha arrollado a varias personas en el } \\
\text { puente de Westminster y } \\
\text { - Luego ha apuñalado a un policía junto al } \\
\text { Parlamento británico } \\
\text { - } 4 \text { personas han muerto en el ataque, incluidos el } \\
\text { policía y el agresor } \\
\text { - El ataque deja al menos } 20 \text { heridos } \\
\text { - Han evacuado a la primera ministra| } \\
\text { MARzo 22, } 2017 \text { 21:41 } \\
\text { Ahí arriba tienes lo esencial. La policía ha retenido durante horas a quienes estaban dentro del Parlamento. } \\
\text { Algunos reporteros han sido testigos del ataque. }\end{array}$ \\
\hline
\end{tabular}




\begin{tabular}{|c|c|}
\hline @usuario & ¿Por ejemplo? \\
\hline$@$ politibot & $\begin{array}{l}\text { Shouts of "ambulance, ambulance!" could be heard. There was no sign of } \\
\text { movement from the police officer who had been attacked or from the assailant, } \\
\text { lying barely } 10 \text { meters further down the cobbled entrance to parliament. } \\
\text { Within } 10 \text { minutes, an air ambulance had landed on the grass of parliament } \\
\text { square, next to the statue of Winston Churchill. Throughout this time, police } \\
\text { officers had been treating their downed colleague. } \\
\text { Around the corner on Westminster Bridge, a car lay rammed into the side of the } \\
\text { railings which wrap around Westminster Palace after hitting several people. } \\
\text { Esas frases están sacadas del artículo de Tom McTague, reportero de Político ¿Quieres leerlo entero? }\end{array}$ \\
\hline @usuario & Sí \\
\hline @politibot & $\begin{array}{l}\text { http://www.politico.eu/article/terror-beneath-my-window/ } \\
\text { POLITICO } \\
\text { Westminster attack: Terror beneath my window } \\
\text { POLITICO reporters witness terror incident in UK parliament. } \\
\text { Algunas personas han sido evacuadas a la Abadía de Westminster. Esta foto la colgó en Twitter Michael } \\
\text { German, miembro de la Cámara de los Lores. Tengo una pregunta de Trivial. Uno de estos personajes } \\
\text { célebres no está enterrado en Westminster. ¿Sabes quién es? }\end{array}$ \\
\hline @usuario & Dickens \\
\hline$@$ @politibot & Error. La respuesta correcta era Thatcher \\
\hline @politibot & $\begin{array}{l}\text { Buenas tardes, human@. La primera ministra británica, Theresa May, se ha dirigido al Parlamento por } \\
\text { primera vez después del ataque de Londres. A continuación te cuento lo que ha dicho. }\end{array}$ \\
\hline
\end{tabular}

Fuente: https://web.telegram.org/\#/im?p=@Politi_bot

De acuerdo al cuadro no 9, es perceptible la conversación de Politibot con los usuarios en un tono cercano y con un discurso dialógico. Y es que, muchos de ellos, se identifican como "fan" del bot por difundir noticias de utilidad con contenido propio y/o con información contrastada de los principales medios. También, es posible señalar la presencia de elementos ilustrativos en la distribución de la información (gráficos e imágenes), así como enlaces para profundizar en la información remitida. 


\subsubsection{Cobertura de Politibot e interactuación emocional con la audiencia}

Para recoger la opinión de la audiencia sobre la cobertura política del bot y la conexión que establece con el usuario, hemos elegido el tema de las elecciones generales de los Países Bajos llevadas a cabo el 15 de marzo de 2017. Para la recogida y posterior análisis de la información, hemos considerando el periodo temporal del 8 al 23 de marzo (teniendo en cuenta un margen entre 7 y 8 días antes y después, respectivamente), como ya se ha explicado en el apartado de acotaciones metodológicas.

En relación a la cobertura del bot sobre las elecciones en Holanda, Eduardo Suárez (2017) explica:

“Teníamos allí a dos de nuestros desarrolladores, que viven en Leiden. Uno de ellos nos hizo llegar imágenes y nos contó detalles de la campaña. Eso nos ayudó mucho a conocer lo que estaba ocurriendo más allá de lo que decían los medios en español y en inglés. El bot, hizo una cobertura en los canales que tenemos abiertos por ahora. Grabamos un episodio de nuestro podcast que tuvo más de 3.000 descargas. Seguimos el minuto a minuto por Twitter, donde durante el mes de marzo hemos superado dos millones de impresiones e hicimos un envío cada día en nuestro bot de Telegram y Facebook Messenger".

Para recoger las opiniones de los usuarios, la herramienta utilizada ha sido Twitter porque los usuarios establecen a través de Telegram un contacto privado, pero a la hora de dar su opinión lo hacen en espacios que favorecen las conductas individuales y colectivas, en todo caso, ciudadanas, como es el caso del microblogging.

Los usuarios reconocen que la información ha sido completa, precisa y sin sesgo político. Asimismo, han valorado la presencia de elementos ilustrativos (gráficos/imágenes) y multimedia (podcast, vídeo y gifs) para complementar la información. Los datos arrojan que, el 24,52\% (13) respecto al 100\% (53) se muestra muy satisfecho, el 66,03\% (32) satisfecho, el 13,20\% (7) normal y el 1,88\% (1) poco satisfecho. Como ejemplo, hemos recogido los siguientes tweets de los usuarios sobre la información proporcionada por el bot referente a las elecciones generales de Holanda.

Cuadro 10: Opiniones de los usuarios sobre el servicio de Politibot

\begin{tabular}{|l|l|l|}
\hline Usuario & Fecha & Mensaje \\
\hline @AlxandraGil & 23 de Marzo & $\begin{array}{l}\text { "Mis días no serían lo mismo sin @politibot. Charlad con él, Informa con precisión, } \\
\text { con un toque de humor, y a veces te hace así ” }\end{array}$ \\
\hline @Imartinaguirre & 16 de Marzo & $\begin{array}{l}\text { "Que cantidad de información tiene @politibot sobre las elecciónes holandesas!! Muy } \\
\text { fan de los gráficos”. }\end{array}$ \\
\hline @raulbroto & 16 de Marzo & $\begin{array}{l}\text { "Yo ya sabía que Wilders no iba a poder gobernar de todas formas, gracias a las “Char- } \\
\text { las" con @politibot! Gracias!” }\end{array}$ \\
\hline @mdetoroh & 16 de Marzo & $\begin{array}{l}\text { "Lo han vuelto a hacer. Enorme cobertura de @politibot en \#paises bajos citando } \\
\text { fuentes y colegas" }\end{array}$ \\
\hline
\end{tabular}




\begin{tabular}{|l|l|l|}
\hline @mcarvajal_ & 15 de Marzo & $\begin{array}{l}\text { Acabo de chatear con @politibot sobre las elecciones holandesas: información, ágil y } \\
\text { con gracia. Si es bueno, el periodismo se abre paso" }\end{array}$ \\
\hline @AlxandraGil & 15 de Marzo & $\begin{array}{l}\text { "A mí @politibot ya me explicó ayer lo que pasaría hoy con \#Wilders. Con gráficos y } \\
\text { gifs. ¿Es para enamorarse o no? }\end{array}$ \\
\hline @mariadelamiel & 15 de Marzo & $\begin{array}{l}\text { "Me declaro \#muyfan de @politibot Si queréis sabes la última hora de la actualidad } \\
\text { política, no lo dudéis" }\end{array}$ \\
\hline @Wiklerman15 & 15 de Marzo & "Cigras de las elecciones en \#Holanda por @politibot” \\
\hline @Mariablanco_ & 14 de Marzo & $\begin{array}{l}\text { "Mi imprescindible de hoy es para @politibot, el periodista robot’ que ofrece informa- } \\
\text { ción, multimedia e interactividad". }\end{array}$ \\
\hline @AlejandraArizaB & 11 de Marzo & $\begin{array}{l}\text { "Interesantísimo podcast de @politibot a propósito de las elecciones en Países Bajos } \\
\text { el próximo 15 de marzo" }\end{array}$ \\
\hline
\end{tabular}

Fuente: Elaboración propia con datos extraídos de https://twitter.com/politibot?lang=es

Es perceptible la satisfacción que muestran los usuarios por el servicio que le ofrece Politibot en Telegram y que se puede apreciar en el cuadro $\mathrm{n}^{\circ} 10$. Otro aspecto a destacar, es la conectividad emocional que pueda existir entre el bot y la audiencia en relación al uso del mismo y que es posible determinar a través de la conducta de aceptación o rechazo. Y es que, el comportamiento se limita a la conexión entre estímulo y respuesta en relación a la información recibida que "adquiere relevancia por el valor otorgado a la intensidad de la transmisión del mensaje, es decir, a la presencia de los elementos emotivos" dentro de este y que se realiza por las vías neurológicas del cerebro por medio de la experiencia (Sánchez Gonzales, 2007: 545).

La emoción "es el resultado de dos tipos de herencias, cultural y biológica, más aún, cuando tiene correlación directa con nuestros objetivos y creencias como forma de existencia humana". El usuario es un ser social por naturaleza y por tanto, construye su realidad dentro de un contexto de interactuación donde las emociones cobran sentido y cambiarían el mismo "según el significado que le demos dentro del contexto de interactuación" (Sánchez Gonzales, 2012).

Para identificar las emociones que despierta el bot por medio de la conversación, hemos tenido en cuenta frases o palabras con fórmulas de cortesía, empleo del lenguaje figurado y la presencia o ausencia de las emociones (Goleman, 1996) y (Bisquerra, 2000). Los datos arrojan 154 conversaciones respecto a la cobertura del boty el 53,89\% (83) de interactuaciones hacen uso del lenguaje figurativo. Los usuarios emplean con el 31,32\% (26) metáforas, con el 25,30\% (21) hipérbole, con el 19,27\% (16) personificación, con el 13,25\% (11) símil e ironía con el 4,81\% (4). Lo que supone que el 46,10\% (71) es un lenguaje neutro (no establece ninguna reacción). Respecto a las emociones que establecen esa conexión entre el boty el usuario, del $100 \%$ (138) de las conversaciones sobre el servicio de noticias, el 88,41\% (122) son positivas el $11,59 \%(16)$ negativas. En ambos casos, hemos considerado el orden sintáctico y elementos afectivos y emocionales que componen el texto. 
También, hemos identificado las siguientes emociones positivas. El amor se sitúa en primer lugar por encima del resto con el 45,90\% (56), con respecto al 100\% (122). Nos referimos a la confianza (25\%), empatía (21,43\%), simpatía (10,71\%), aceptación (5,36\%), afinidad (8,93\%), respeto (5,36\%), adoración (7,14\%), enamoramiento (12,5\%) y gratitud (3,57\%). En segundo lugar se posiciona la felicidad con el 22,95\% (28), y, entre ellas, gozo (3,57\%), tranquilidad (28,57\%), satisfacción $(42,86 \%)$, bienestar $(17,85 \%)$ y placidez $(7,14 \%)$. En tercer lugar, la alegría con el $18,03 \%$ (22) y de forma concreta: satisfacción (40,91\%), entusiasmo (18,18\%), euforia (4,54\%), alivio (13,64\%), regocijo (9,09\%) y diversión (13,64\%). En cuarto lugar, el humor con el 9,84\% (12) a través de la risa (se ha utilizado expresiones y emoticons) y finalmente, las emociones ambiguas con el 3,28\% (4): sorpresa y esperanza, ambas con el (50\%).

Por otro lado, las emociones negativas que se han localizado son, en primer lugar, la ansiedad con el 43,75\%, respecto al $100 \%$ (16), y, entre ellas: desesperación, inquietud y nerviosismo con igual porcentaje (14,29\%), y consternación (57,14\%). Asimismo, la tristeza con el $43,75 \%$ y en concreto: aflicción y pena (28,57\%) en ambos casos, así como desconsuelo, pesimismo y melancolía con similar porcentaje (14,29\%). Finalmente, la aversión con el 6,25\%, y, entre ellas, la antipatía. Estas emociones se manifiestan en la interactuación pero hacen referencia al sentimiento hacia la noticia y no propiamente a la cobertura del bot. Al respecto, a modo de ejemplo, hemos extraído el siguiente cuadro (nº11).

Cuadro 11: Interacción emocional con la audiencia

\begin{tabular}{|c|c|c|}
\hline Usuario & Fecha & Mensaje \\
\hline @SalvaBenasayag & 20 de Marzo & “El @politibot es mejor que un terapeuta”. \\
\hline @cygenta & 17 de marzo & “Se están equivocando si aún no conversan con @politibot en \#telegram”. \\
\hline$@$ @_molins & 16 de marzo & “@politibot estás bien? Tu silencio me tiene preocupado. No me has dicho nada más :(”. \\
\hline @ccandidop & 16 de marzo & $\begin{array}{l}\text { "Confieso que me he enamorado de un robot. Todo lo que sé sobre las \#eleccionesNL me lo ha } \\
\text { contado él.@politibot”. }\end{array}$ \\
\hline @JaviSkan & 16 de marzo & $\begin{array}{l}\text { "@politibot está dormido todavía. Pero me cuenta cosas de Holanda de buena mañana, así que } \\
\text { se le perdona todo". }\end{array}$ \\
\hline$@$ mlopca & 15 de marzo & “@politibot thanks! Si te pasas por Barcelona te dejo los enchufes de mi casa para que te cargues”. \\
\hline @NilGarciaE & 15 de marzo & "Amor político, @politibot" \\
\hline @tammylidon & 14 de marzo & $\begin{array}{l}\text { "No puedo ser más \#FanTotal del \#Telegram de @politibot .Por fin un robot que entiende mis } \\
\text { sentimientos politológicos" }\end{array}$ \\
\hline @AlxandraGil & 13 de marzo & @politibot ha ligao. Arte. \\
\hline @juanfont & 11 de marzo & $\begin{array}{l}\text { "Los de @politibot son los primeros términos de uso que molan en la historia de la Humanidad, } \\
\text { delUniverso y de Todo. http://telegra.ph/Politibottérminos-de-uso-03-05" }\end{array}$ \\
\hline @Naeri & 9 de marzo & $\begin{array}{l}\text { "Coloca un @politibot en tu vida y nunca más te sentirás solo. Al menos tengo alguien que me } \\
\text { habla cada mañana...” }\end{array}$ \\
\hline
\end{tabular}

Fuente: Elaboración propia con datos extraídos de https://twitter.com/politibot?lang=es 
Si bien es cierto, Politibot en el mes de junio de 2016 (mes de su creación) alcanzó 8.400 suscriptores, a partir del 8 de marzo, cuando el bot se puso nuevamente en marcha, obtuvo 3.210 nuevos usuarios y en menos de treinta días, así nos aseguró Suárez (2017). Su intención es cubrir los acontecimientos políticos que sucedan en España, Europa y EE.UU., como las elecciones de Francia y las primarias del PSOE usando firmas de encuestas y gráficos propios y de otros medios. "Siempre citando el origen, claro".

No cabe duda de la relación empática que establece el bot con la audiencia en Telegram y que es posible corroborar a través del cuadro no 11 . A ello, le sumamos que Twitter es un espacio que reduce el estrés debido a que el "cerebro parece ver la interacción virtual como si las personas estuvieran junto a nosotros" (Zak, 2010).

A la luz de los datos hallados sobre la cobertura política (elecciones en Holanda) por parte del boty la conexión emocional con el usuario, respectivamente, el cuadro 12 muestra en términos de porcentaje que del $100 \%$ (283) interacciones o respuestas por parte de los usuarios (retweet), y teniendo en cuenta el periodo temporal establecido, el 54,41\% (154) tiene que ver con la cobertura general sobre noticias difundidas por el bot, el 18,72\% (53) sobre las elecciones de Holanda, teniendo en cuenta que, el 66,03\% (35) se corresponde con el 15 de marzo (día de las elecciones). Asimismo, el 26,85\% (76) cerca de la mitad, con respecto a la cobertura sobre la actualidad política, proviene de la conexión emocional que establece el bot con el usuario. No obstante, es posible señalar que el 23,68\% (18) de dicha interactuación se produce con la cobertura sobre el atentado en Londres (23 de marzo). Datos que arrojan la preferencia y apuesta de los usuarios por la información personalizada y con valor añadido.

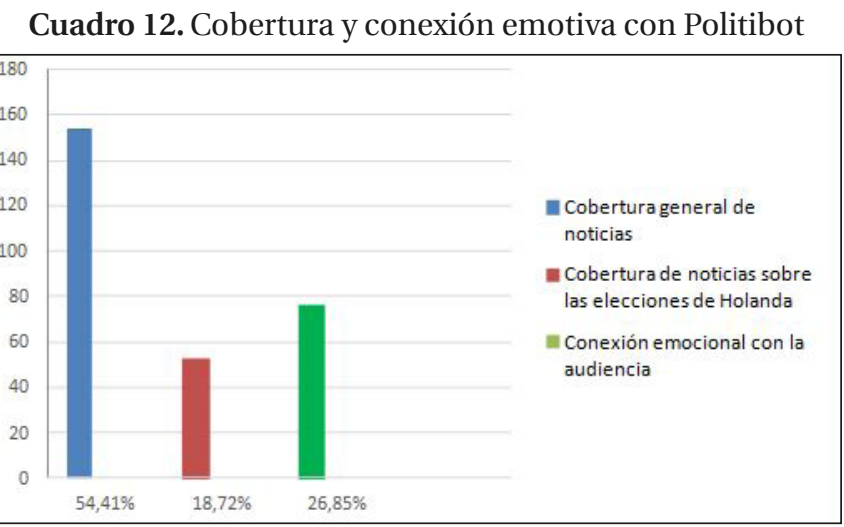

Fuente: Elaboración propia 
Si bien es cierto, Politibot en el mes de junio de 2016 (mes de su creación) alcanzó 8.400 suscriptores, meses después, en marzo (a partir del 8), cuando el bot se puso nuevamente en marcha, obtuvo 3.210 nuevos usuarios hasta el 29 del mismo mes y en menos de treinta días, así nos aseguró Suárez (2017). Su intención es cubrir los acontecimientos políticos que sucedan en España, Europa y EEUU, como las elecciones de Francia y las primarias del PSOE usando firmas de encuestas y gráficos propios y de otros medios. "Siempre citando el origen, claro".

\section{Conclusiones}

Frente a la sobredosis informativa con la que tenemos que convivir por parte de los medios, se establecen nuevas fórmulas personalizadas y de gestión de la información a través de aplicaciones de mensajería instantánea; con un discurso próximo y recursos que despiertan el interés y hacen que el usuario conecte emocionalmente con ellas. Los medios están apostando por la creación de un canal en Telegram con presencia activa en la aplicación. Es el caso de la agencia de noticia Efe y medios de comunicación como: eldiario.es, El País, El Mundo, elEconomista, El Español, El Periódico, El Confidencial, CTXT, Cambio16, Cuarto Poder, El Jueves, OKdiario, Público y Vozpópuli.

En este contexto, aparece Politibot, el primer bot en España que ofrece servicios de noticias sobre la actualidad política en Telegram y con gran aceptación por parte de la audiencia. En solo 21 días (del 8 al 29 de marzo) ha registrado 3.210 nuevos usuarios. De igual forma, su éxito también se corrobora con el 73,13\% de respuestas por parte de los usuarios que hacen referencia a la calidad informativa. Es por ello que, el $90,55 \%$ se muestra satisfecho $(66,03 \%$ y muy satisfecho $(24,52 \%)$ con la distribución de la información por parte del bot. La cobertura de información personalizada, ágil, precisa y sin sesgo político debido a sus fuentes (propias y externas) es lo que caracteriza al bot. Asimismo, complementa la información con gráficos, imágenes, gifs y podcast. Este último tuvo gran acogida tras difundir el episodio referido a las elecciones de Holanda con más de 3.000 descargas y por otro lado, con dos millones de impresiones en Twitter, durante el mes de marzo, tras seguir minuto a minuto lo que sucedía en los Países Bajos, Suárez (2017). Como muestra, algunas de las respuestas de los usuarios: "Acabo de chatear con @politibot sobre las elecciones holandesas: informativo, ágil y con gracia. Si es bueno, el periodismo se abre paso" (r1); "Lo han vuelto a hacer. Enorme cobertura de @ politibot en \#paisesbajos citando fuentes y colegas" (r2).

También, es posible calificar la interactuación con la audiencia como ejemplar y que a la fecha, ningún medio ha experimentado a pesar de la demanda del usuario. El 53,89\% de esas interactuaciones hacen uso del lenguaje figurativo y el $46,10 \%$ alude a un lenguaje neutro que no genera reacciones. Los mensajes de texto publicado en Twitter revelan que el $88,41 \%$ de las emociones son positivas, respecto al 11,59\% que son negativas. En el primer caso, predominan por parte del usuario la satisfacción (83,77\%), confianza (25\%) y empatía (21,43\%), y, respecto a las emociones negativas, consternación $(57,14 \%)$ y aflicción $(28,57 \%)$. Estos últimos porcentajes representan las emociones que han sido suscitadas por 
la noticia en sí misma y no por la cobertura del bot sobre las elecciones en Holanda. Datos que reflejan que el usuario ha establecido una relación más estrecha, emotiva y de identificación con el robot, así lo corrobora el 26,85\%, es decir, cerca de la mitad de la participación sobre el servicio de noticias del bot, por tanto, es un dato significativo. Como muestra, algunas de las respuestas de los usuarios: “@charolarrea: @politibot está suceptible” (r1), "el @politibot me acaba de decir que molo. Con la fama de ligón que lleva no sé cómo tomármelo... guiño guiño...@AlzandraGil” (r2).

En la era líquida, impersonal y con infoxicación, Politibot es la respuesta de los usuarios que quieren estar informados sobre una temática específica y sentirse, en cierta forma, atendidos de manera personalizada y personificada. La información especializada cobra nuevamente especial interés, en este caso, sobre política nacional e internacional sin mencionar que el contenido y la calidad de la información sigue siendo el rey del formato. Por otro lado, con el bot se erige una excelente oportunidad para recoger datos fiables por parte de los usuarios y que los medios de comunicación, en muy corto plazo, aprovecharán la ventaja que ofrece este escenario. Por todo ello, podríamos decir que los bots no suponen un peligro para el periodismo, sino una oportunidad para su desarrollo.

\section{Referencias bibliográficas}

233grados.com/La Información (2014): "The Guardian lanza en EE.UU. un periódico en papel, gratuito y hecho por un robot”. En 233grados.com (blog de La Información), 17 de abril de 2014 http://233grados.lainformacion.com/ blog/2014/04/the-guardian-lanza-en-eeuu-un-peri\%C3\%B3dico-en-papel-gratuito-y-hecho-por-un-robot.html [Consultado el 07/04/2017].

Bauman, Z. (2002): Modernidad líquida. México D. F.: Fondo de Cultura Económica.

Bisquerra, R. (2000): Educación emocional y bienestar. Barcelona: Praxis.

Bunz, M. (2010): “In the US, algorithms are already reporting the news”. En The Guardian, 30 de marzo de 2010. https://www.theguardian.com/media/pda/2010/mar/30/digital-media-algorithms-reporting-journalism [Consultado el 07/04/2017].

Clerwall, Ch. (2014): "Enter the Robot Journalism. Users' perception of automated content”. Journalism Practice, vol 8, 2014. Issue 5: Future of Journalism: in an age of digital media and economic uncertainty, pp. 519-531. http://dx.doi.org/1 0.1080/17512786.2014.883116.

Centro de Estudios Sociológicos (2017): Barómetro de febrero. http://www.cis.es/cis/opencms/ES/index.html [Consultado el 06/04/2017].

Dörr, Konstantin N. (2015): "Mapping the field of Algorithmic Journalism”. Journal Digital Journalism, volume 4, 2016, Issue 6, pp. 700-720. http://dx.doi.org/10.1080/21670811.2015.1096748 [Consultado el 08/03/2017]. 
El País (2014): “El País lanza 'Verne', la web que explora el lado asombroso de Internet”. El País, 24 de septiembre de 2014. http://sociedad.elpais.com/sociedad/2014/09/24/actualidad/1411574486_832029.html(07/04/2017) [Consultado el 08/02/2017 ].

El País (2016): "Llévate EL PAÍS a la playa con nuestro bot”. El País, qué hacemos, 20 de julio de 2016. http://blogs.elpais.com/que-hacemos/2016/07/11\%C3\%A9vate-el-pa\%C3\%ADs-a-la-playa-con-nuestro-bot.html [Consultado el 07/04/2017].

Emmerentze, E. (2014): “El autor más prolijo de Wikipedia”. The Wall Street Journal, 20 de julio de 2014. http:/ /lat.wsj.com/ articles/SB10001424052702304907404580035753339038056 [Consultado el 07/04/2017].

Fernández, R. (2004): Evaluación psicológica. Conceptos, Métodos y Estudio de casos. Madrid: Pirámide.

Gibbs, S. (2016): "Chatbot lawyer overturns 160,000 parking tickets in London and New York". https://www.theguardian.com/technology/2016/jun/28/chatbot-ai-lawyer-donotpay-parking-tickets-london-new-york [Consultado el 08/04/2017].

Goleman, D. (1996): Inteligencia emocional. Barcelona: Kairos.

Gani, A y Haddou, L. (2014): “Could robots be the journalists of the future?”. The Guardian, 16 de marzo de 2014. https:// www.theguardian.com/media/shortcuts/2014/mar/16/could-robots-be-journalist-of-future [Consultado el 07/04/2017].

González, M. (2011): “The Guardian crea un bot que automatiza las búsquedas sobre la actualidad vía Twitter”. Genbeta.com, 29 de octubre de 2011. https://www.genbeta.com/redes-sociales-y-comunidades/the-guardian-crea-un-bot-que-automatiza-las-busquedas-sobre-la-actualidad-via-twitter [Consultado el 07/04/2017].

Johri, A.; Han, E. (Sam) y Mehta, D. (2016): "Domain Specific Newsbots Live Automated Reporting Systems involving Natural Language Communication”. Computation + Journalism Symposium 2016. University of Standford, California, EE.UU. https://journalism.stanford.edu/cj2016/files/Newsbots.pdf [Consultado el 08/03/2017].

Manchón, E. (2016): “Por qué los chatbots matarán tu web, tu app y quizás también a Google”. Eduardomanchon.com, 6 de abril de 2016. https:/ / eduardomanchon.com/por-qu\%C3\%A9-los-chatbots-matar\%C3\%Aln-tu-web-tu-app-y-quiz\%C3\%A1s-tambi\%C3\%A9n-a-google-6c612aac4bfc\#.24hy6d2bv [Consultado el 07/04/2017].

Martínez Carazo, P. (2006): "El método de estudio de caso: estrategia metodológica de la investigación científica”. Pensamiento \& Gestión, 20, pp. 165-193.

Lemelshtrich Latar, N. (2015): “The New World of Transitioned Media”. Einav, Gali (Ed.) The Economics of Information, Communication, and Entertainment. Digital Realignment and Industry Transformation. Sprinter, 65-80. https://link. springer.com/chapter/10.1007/978-3-319-09009-2_6\#page-1 [Consultado el 08/03/2017]. 
Llop, P. (2016): "Periodismo bot: qué puede pasar, cómo puede pasar". Medium.com, 3 de mayo de 2016. https://medium. com/la-maquina-de-las-noticias/periodismo-bot-qu\%C3\%A9-puede-pasar-c\%C3\%B3mo-puede-pasar-185936ab6d2c\#.7gy989mqy [Consultado el 07/04/2017].

Lokot, T. y Diakopoulos, N. (2015): "News Bots. Automating news and information dissemination on Twitter". Journal Digital Journalism, volumen 4, 2016. Issue 6, 682-699. http://dx.doi.org/10.1080/21670811.2015.1081822 [Consultado el 08/03/2017].

Morán, K. (2016): “NBC News presentó un bot de política para Facebook Messenger”. Clases de Periodismo, 12 de octubre de 2016. http://www.clasesdeperiodismo.com/2016/10/12/nbcnews-presento-un-bot-de-politica-para-facebook-messenger/ [Consultado el 07/04/2017].

O’Reilly, T. y Battelle, J. (2009): “Web Squared: Web 2.0 Five Years On”. En Web 2.0 Submmit, Special Report. O’Reilly Media Inc./ Techweb. http://assets.en.oreilly.com/1/event/28/web2009_websquared-whitepaper.pdf [Consultado el 08/03/2017].

Pachico, E. (2016): “Lo que necesitas saber para crear un bot de noticias”. IJnet, 2 de noviembre de 2016. https://ijnet.org/ es/blog/lo-que-necesitas-saber-para-crear-un-bot-de-noticias [Consultado el 07/04/2017].

Pérez, A. (2017): "El Vocento Media Lab lanza InfoEsquí, un experimento de "periodismo-robot" para la temporada de nieve”. Periodista Digital, 23 de febrero de 2017. http://www.periodistadigital.com/periodismo/internet/2017/02/23/ el-vocento-media-lab-lanza-infoesqui-un-experimento-de-periodismo-robot-para-la-temporada-de-nieve.shtml [Consultado el 07/04/2017].

Politibot (2017): Politibot: términos de uso. http://telegra.ph/Politibot-términos-de-uso-03-05 [Consultado el01/03/2017].

Sainz, M./Vocento Media Lab (2016): “Los bots han llegado... ipara quedarse?”. Medium.com, 16 de julio de 2016. https:// medium.com/@VocentoLab/los-bots-han-llegado-para-quedarse-214448cbc442 [Consultado el 07/04/2017].

Salaverría, R. (2015). "Los 'labs' como fórmula de innovación en los medios”. El Profesional de la Información, vol. 24, núm. 4, pp. 397-404.

Sánchez Gonzales, H. y Sobrados León, M. (2008): “El paradigma del rol identitario-próximo al ciudadano en el nuevo formato: ‘Tengo una pregunta para usted' de Televisión Española”. En Pérez, R; Núñez, S. y García, A. (coord.). Comunicacion, Identidad y Género. Vol. 1. Madrid: Fragua, pp. 201-214.

Sánchez Gonzales, H. (2012): "Los dispositivos móviles y la conectividad emocional con el usuario". TechPuntoCero. http://www.techpuntocero.com/2012/05/15/los-dispositivos-moviles-y-la-conectividademocional-con-el-usuario

Sánchez Gonzales, H. (2007): "El estado emocional del miedo como paradigma discursivo en el asenso del político desde la receptividad del mensaje”. Estudios sobre el mensaje periodístico. 13, pp. 543-556. http://dx.doi.org/10.5209/rev_ ESMP.2007.v13.13051. 
Sánchez Gonzales, H. y Méndez Muros, S. (2013): "Nuevos medios, nuevos perfiles y conectividad emocional con la audiencia: los periodistas en la praxis profesional” CIC Cuadernos de Información y Comunicación. Vol. 18. Núm. 1, pp. 133-149.

Sánchez González, M. (2013): “Movilidad y participación”. En Cabrera González, M. Ángeles (coord.). Evolución de los cibermedios. De la convergencia digital a la distribución multiplataforma. Madrid: Fragua (Biblioteca Ciencias de la Comunicación), pp. 129-143.

Sánchez González, M. (2011). "Los smartphones como herramienta para el periodismo móvil: potencial y tendencias de uso por profesionales de la información, usuarios y empresas periodísticas”. Comunicación presentada en III Congreso Latina de Comunicación Social. Universidad La Laguna, 5, 7 y 9 de diciembre de 2011. En Actas, en CD-rom, del III Congreso.

http://www.revistalatinacs.org/11SLCS/actas_2011_IIICILCS/076.pdf [Consultado el 08/03/2017].

Suárez, E. (2016): Entrevista realizada en Sinercom (Networking entre proyectos universitarios y empresas) organizado por el Laboratorio de Proyectos en Comunicación (LabProCom). Universidad de Sevilla.

Suárez, E. (2017): Entrevista sobre la cobertura informativa de las elecciones en los países bajos, realizada por Hada Sánchez. Universidad de Sevilla.

Thurman, N.; Dörr, K. y Kunert, J. (2017):"When Reporters Get Hands-on with Robo-Writing. Professionals consider automated journalism's capabilities and consequences”. Journal Digital Journalism, vol. 5 (2017). Special Issue: Journalism, Citizenship and Surveillance Society, 1-20.

http://www.tandfonline.com/doi/full/10.1080/21670811.2017.1289819 [Consultado el 08/03/2017].

van der Kaa, H. y Krahmer, E. (2014): “Journalist versus news consumer: The perceived credibility of machine written news". Proceedings of the Computation+Journalism conference New York. https://pure.uvt.nl/portal/files/4314960/c [Consultado el 08/03/2017].

van Dalen, A. (2012): "The algorithms behind the headlines. How machine-written news redefines the core skills of human journalists”. Journalism Practice, vol. 6, issue 5-6 The Future of Journalism 2011: Developments and Debates. http:// dx.doi.org/10.1080/17512786.2012.667268 [Consultado el 08/03/2017].

VV.AA. (2016): La Sociedad de la Información en España. SiE16. Fundación Telefónica/Ariel.http://www.fundaciontelefonica.com/arte_cultura/publicaciones-listado/pagina-item-publicaciones/itempubli/558/ [Consultado el 08/03/2017]. Zak, P. (2010): Social Networking Affects Brains Like Falling in Love. Fast Company, 147, (julio/agosto). http://www.fastcompany.com 